\title{
464 - SURVEY OF THE ADEQUACY OF HOSPITAL DISCHARGE LETTERS FROM OLDER ADULT MENTAL HEALTH (OAMH) WARDS IN A SCOTTISH HEALTH REGION
}

Gary Stevenson ${ }^{1}$ Sharon Munro $^{1}$, Connor McIntyre ${ }^{1}$ \& Stephen Foster ${ }^{2}$

${ }^{1}$ NHS Fife, Fife, Scotland (gstevenson@nhs.net), ${ }^{2}$ SE Scotland Psychiatric Rotation, Scotland

Background: One form of communication deficiency leading to patient harm is failure to keep colleagues informed and to share appropriate levels of clinical information. The production of discharge letters is a clinical and professional requirement, deficiencies of which contribute to clinical risks, while failure to observe standards may be a focus of medico-legal enquiry.

Objectives: To examine the adequacy of clinical discharge letters from the OAMH inpatient wards in one Scottish Health region (Fife, population 370,000) against the 14-day discharge policy, with focus on medication advice and follow-up arrangements.

Methods: All discharge notifications from the five $\mathrm{OAMH}$ wards were examined retrospectively against the electronic records and case-files for the 7-month period ending $31^{\text {st }}$ January 2020.

Results: 169 discharge notifications inclusive of 14 deaths were reduced to 123 after excluding brief inter-ward transfers. Female:male ratio of 1.05:1; average age 77 (range 60-99) years, average inpatient duration 120 (range 2-934) days. There was no identified discharge letter in 20.3\%. Direct admissions from Care Homes died more often (30\%) than those admitted directly from home (2\%), presumably a reflection of greater frailty. $29 \%$ patients were discharged to (19\% admitted from) Care Homes. $59 \%$ patients had dementia, $20 \%$ an affective disorder, $7 \%$ a psychotic disorder, with $20 \%$ having multiple diagnoses. Antidepressants were the commonest (49\%) regularly prescribed psychotropic medication on discharge both for those with $(47 \%)$ or without $(52 \%)$ dementia. $32 \%$ of all patients ( $25 \%$ in dementia) were discharged on antipsychotics, often without advice on monitoring, prescribing restrictions or risks. The 98 verified letters took 27 (range 0-168) days to verify, 67\% failing the production-time standard. $53 \%$ discharges had multiple follow-up arrangements. Variabilities were noted in letter production according to the discharge ward (range 53-100\%) and between consultant teams (verification rates 50 $100 \%$ ) where delays ranged 6-109 days and ability to produce letters within the standard ranged from 092\% (average 33\%).

Conclusions: There appear significant failings in the timely transfer of clinical details between OAMH inpatient services and primary care services in this region that require intervention to minimise clinical risk and maximise patient safety. There were identified factors that are amenable to quality improvement. 